\title{
Note on the vegetation of the mounts of tlemcen (Western Algeria): Floristic and phytoecological aspects
}

\author{
Brahim Babali, Abderrahmane Hasnaoui, Nadjat Medjati, Mohamed Bouazza \\ Laboratory of Ecology and Management of the Natural Ecosystems, Department of Ecology and Environment, Aboubakr Belkaid \\ University, Tlemcen, Algeria; 'Corresponding Author: miharb_babali@hotmail.fr \\ Received 5 February 2013; revised 8 May 2013; accepted 8 August 2013 \\ Copyright (C) 2013 Brahim Babali et al. This is an open access article distributed under the Creative Commons Attribution License, \\ which permits unrestricted use, distribution, and reproduction in any medium, provided the original work is properly cited.
}

\begin{abstract}
Of the four national hunting reserves in Algeria, the Mounts of Tlemcen Moutas reserve

http://reservebio-tlm.com, characterized by a large area, reliefs and a specific climate, implies significant floristic and faunistic richness. Currently, the coexistence of species, such as Quercus faginea subsp. tlemcenensis (DC.) M., Lonicera implexa L., Ruscus aculeatus L., indicates a forest dominant ecological atmosphere, although the region has experienced repeated fires during the 90's. In this research, a phytoecologicaland syntaxonomical analysis is obvious. More than 300 species have been inventoried and indexed in more than $\mathbf{7 0}$ families and this shows the importance of phyto-diversity of the studiedregion. In the analysis of the phyto-ecological parameters, we could notice a regression of the vegetal cover in its diversity.
\end{abstract}

Keywords: Biodiversity; Floristic inventory; Phytoecological; Anthropozoological action; Climate; Moutas; Tlemcen

\section{INTRODUCTION}

The currently developed methods of biodiversity extinction have large uncertainties but all converge on acceleration whatever would be the economic models. To assess the loss of biodiversity, we worked on the disappearance and fragmentation of plant life media (inventtory of natural habitats).

The reserve is particularly sensitive in terms of plant diversity, it underwent in the past human pressures and significant fires. The ecological landscape includes different habitats moving to a scrub, with considerable variations.
The knowledge of this dynamic and this floristic inventory is an important research path for us. Analyses of biodiversity lead in particular to show that the maximum biodiversity is not in the primitive forest sensu stricto, but in the moderately man altered spaces [1].

We will discuss this problem here from floristic inventory formed by tree structures and their stages of degradation as it is at this level that they can be analyzed.

The vegetation of the national parks and natural reserves in the Mediterranean basin have been studied by many authors like Gruber and Sandoz [2]; Véla et al. [3]; Hill and Véla [4]; Ibn Tattouand \& Fennane [5]... and other works in Tlemcen region like those of Benabadji et al. [6]; Mesli et al. [7]; Letreuch-Belarouci et al. [8]; Medjahdi et al. [9] and Bouazza et al. [10].

\section{METHODOLOGY}

Location and structure of Tlemcen hunting reserve:

The study area is located in the western part of Northwest Algeria at about $46 \mathrm{~km}$ as the crow flies from the sea and $26 \mathrm{~km}$ south-west of the city of Tlemcen. The reserve, part of Hafir forest, occupies the highest and most wooded area of the Mounts of Tlemcen. It is located about $34^{\circ} 41^{\prime}$ to $34^{\circ} 49^{\prime}$ north and $01^{\circ} 25^{\prime}$ to $01^{\circ} 35^{\prime}$ west (Figure 1).

It occupies an area of 2156 hain a $15 \mathrm{~km}$ perimeter; it is characterized by typically mountainous reliefs of the Tamaksalet massif with a remarkable difference in altitude. The altitude is between the extreme points from Ras Torriche $1303 \mathrm{~m}$ andthe region of Sidi Messaoud at $1017 \mathrm{~m}$.

It is geographically limited:

- To the east by the town of Aïn Ghoraba;

- In the north-east by the municipality of Sabra;

- To the west and northwest by the municipality of Bouhlou; 


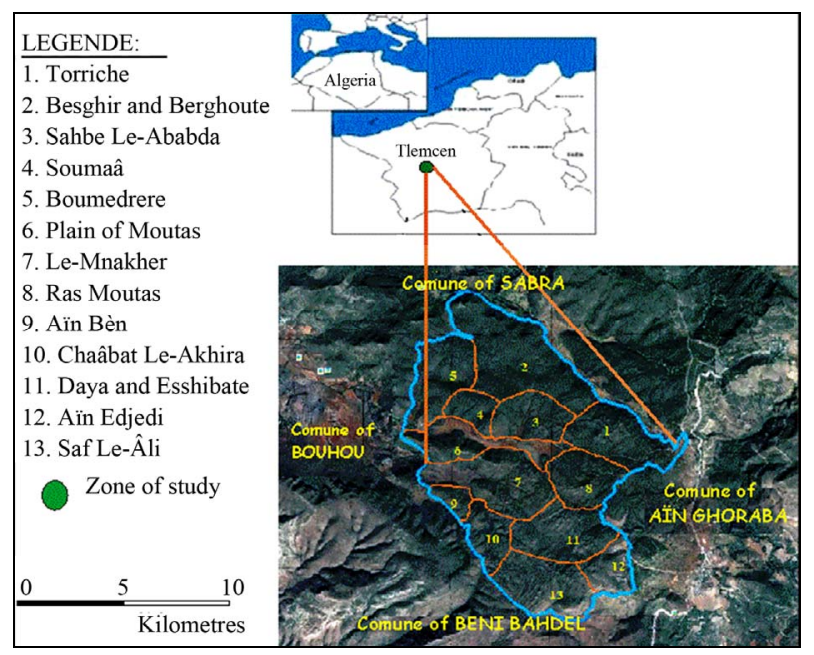

Figure 1. Location map.

- In the south and southwest by the city of Beni Bahdel.

It receives about $500 \mathrm{~m}$ mannual rainfall and shows average temperatures between $6.4^{\circ} \mathrm{C}$ (January) and $26.4^{\circ} \mathrm{C}$ (July and August). It is classified in the cool winter's sub-humid bioclimatic level with a dry season (5 months) which lasts from June to October.

The soils are varied depending on the topography of the region. On the tops, they are not very deep where of-time the bed rock levels. Sometimes deep towards very deep on all along the principal river basins of the hydrographic network.

In the reserve, the main rocks source is sedimentary: calcareous sandstone, sandy limestones, dolomites and marl [11].

The complete list was made from the following works:

Moutas Herbarium which is made up to now more than 220 species recorded by researchers from the laboratory of plant/botanical ecology from the University of Tlemcen,

Field work during 2010, 2011 and 2012, we have completed 70 surveys using conventional techniques and methods of ecology (inventory, minimum area, transect, flora network...).The method of Braun-Blanquet [12], which is expressed by analytical characteristics: abundance, dominance and sociability on a scale of 1 to 5 to help us to do a thorough analysis of the vegetation.

The basic work used for the identification of taxa collected in the field is from the studies done by Quézel \&Santa [13]; Battandier \& Trabut [14]; Valdéset al. [15]; Blanca et al. [16]; Maire [17] and Dobignard [18].

Scientific Name: the word list adopted is that of: Index synonymique et bibliographique de la flore d'Afrique du Nord [18] and synonyms from: Nouvelle flore d'Algérie et des régions désertiques méridionales [13].

\section{RESULTS AND DISCUSSIONS}

Ecological zoning of the main forest groupings.

\subsection{The Oak Forests}

Four major species of the genus Quercus were found in the reserve: Quercus ilex L., Quercus suber L., Quercus coccifera L. and Quercus faginea subsp. tlemcenensis (DC.) Maire and Weiller (=Quercus faginea subsp. Broteroi (Coutinho) A. Camus).

\subsubsection{Evergreen Oak Forest}

Dominates almost all of the reserve and is characterized by the evergreen oak presence (Quercus ilex subsp. ballota (Desf.) Samp) within a 942.4 hectares area.

The major issues, well developed and characterized by a large trunk, are located at the edges of cultivated lands within the reserve where the soil is deep and very rich in organic matter. These characters change as the altitude increases, the soil becomes shallower and bedrock appears at the surface. In addition, North exposures have a significant contribution to water compensation, allowing the taxa development in integrated settlements linked to Quercetea ilicis [6,19].

The distribution of Kermes oak (Quercus coccifera subsp. coccifera L.) is very limited, and in the extreme west and north-west of the reserve close to Tamaksalet (Bouhlou municipamity) and some species are scattered throughout the center of the reserve at Ras Mnakher, Mnakher and Souamaa. These areas are a warmer (xeric).

\subsubsection{Zeen Oak Forest}

The zeen oak (Quercus faginea Lamk) is a deciduous oak of meso-and supra-Mediterranean types [20-22], endemic to the western Mediterranean (Iberian Peninsula, Morocco, Algeria and Tunisia) [23]. It would be represented in the Mounts of Tlemcen by a sub-species: Quercus faginea subsp. tlemcenensis (DC.) M. (Figure 2)

This oak occupies $1 / 5^{\text {th }}$ of the reserve with an area of 428 ha. It is found mainly in the southern and south-

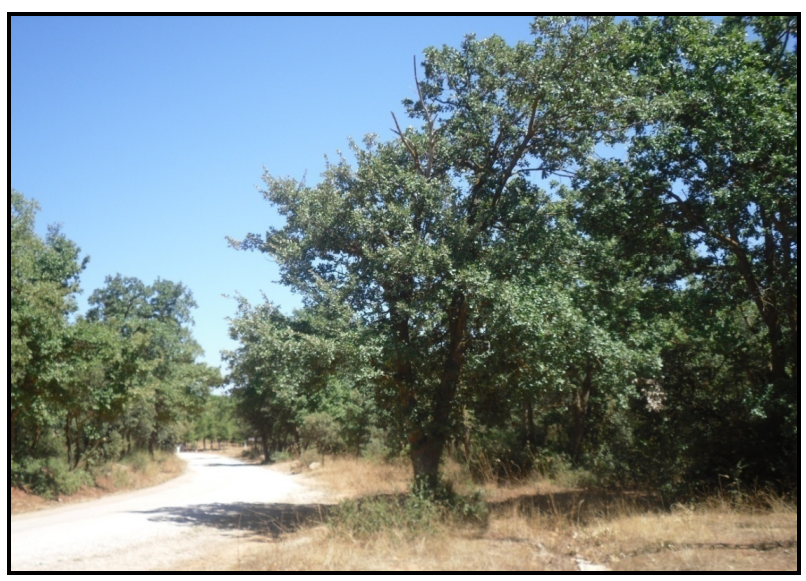

Figure 2. Quercus faginea subsp. tlemcenensis formations at Tor- riche (Moutas). Photo. Babali B. September 2011. 
western part of the reserve at Torriche, Ras Moutas, Mnakher, Chaâbat La'akhra and Aïn Ben. It is also found in the extreme north at Besghirand Boumedrer.

The Tlemcen zeen oak, ranging in size between 5 and $7 \mathrm{~m}$, prefers deep soils and limestone-rich substratewithfresh degraded materials and rare silica. This species exists and dominates the valleys and hollows of the reserve.We can consider that this species benefits from the water compensation, despite drought and this can be explained by compensation edaphic-climatic phenomena [24]. It is practically non-existent or so on the summit where the soil depth is less thick, and even if it does, it is most unusual and with a dwarf size that barely exceeds 2 $\mathrm{m}$.

Among the accompanying taxa are: Cytisus arboreus subsp. Baeticus (Webb) Maire Cytisus villosus Pourret, Hedera algeriensis Hibberd, Ruscus aculeatus L., Smilax aspera L., Viburnum tinus L., Lonicera implexa L., Pistacia terebinthus L., Asplenium ceterach L., Umbilicus rupestris (Salisb.) Dandy, Phillyrea latifolia L. Ampelodesmos mauritanicus (Poiret) Durand \& Schinz...

\subsubsection{Cork Oak Forests}

Representedby the cork oak relics: Quercus suber L. (Figure 3), in a very limited area, which does not exceed 20 ha, they are frequently found in Saf-el-Ali, Aïn Djedi and other relics in Torriche, Boumedrerand Ras Moutas south side and finally a few stalks at Mnakher. Their growth is generally less strong after fire. These species are typical of low-intensity fire regimes, but common in the study area [25-27].

The vegetation associated with these cork oak is: $L a-$ vandula stoechas L., Anagallis arvensis L., Erica arborea L., Arbutus unedo L., Stauracanthus boivinii (Webb) Samp Ampelodesmos mauritanicus (Poiret) Durand \& Schinz, Asparagus acutifolius L., Daphne gnidium L., Cytisus villosus Pourret, Cistus clusii Dunal., Cistus creticus L., Cistus salvifolius L., Cistus ladanifer subsp.

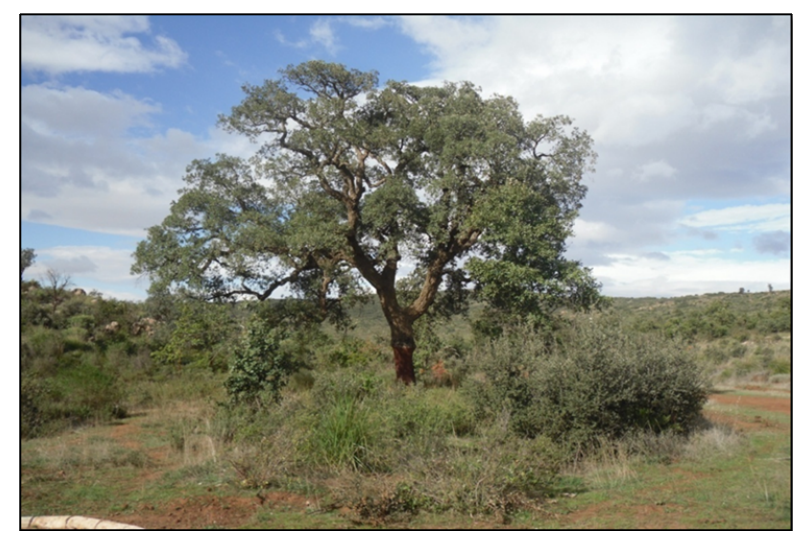

Figure 3. Relic of cork oak. Sahb El Ababda (Moutas). Photo. Babali B. October 2010. mauritianus Pau \& Sennen. These plants prefer siliceous substrates.

\subsection{Conifers}

They are softwood thermophilic with an extremely wide ecological spectrum. We have: Thuja: Tetraclinis articulata (Vahl) Masters (Figure 4).

Endemic to North Africa [28,29] it colonizes areas with low rainfall (300 - $500 \mathrm{~mm}$ ) [30]. This species is slightly represented in the Moutas reserve. It occupies, especially the northwest portion of the reserve: southwestern slopes and the southern slopes of Boumedrer, Ras Mnakhert toward Bouhlou, Aïn Ben Soumaâ and Safel-Ali. It is associated with Pistacia lentiscus L., Chamaerops humilis var. argentea Andrew Globularia alypum L., Macrochloa tenacissima L. (Kunth), Phyleria angustifolia L., Asparagus albus L.

\section{The Juniper: Juniperus oxycedrus Subsp. Oxycedrus}

It is widespread in the reserve with scattered blankets. This indicates the presence of degradation oak stands.

- Other conifers are represented in the form of plantations in the rest area Torriche and near the forest house Boumedrer as Aleppo pine Pinus halepensis Miller, stone pine (Pinus pinea L.) cedar (Cedrus atlantica (Endl.) Carrière) and cypress (Cupressus sempervirens L.)

\subsection{Riverine}

The reserve is surrounded by natural sources: AïnBoumedrer the largest and most common, AïnE-Djedi, Aïn Moutas and Aïn El-Ben. The vegetation, adjacent to these springs and streams, is riparian representing vegetation structure at least partly azonal [31], or indicators of wetlands such as Rubus ulmifolius Schott, Dittrichia viscosa (L.) Greuter, Typha latifolia L., Carex hispi-

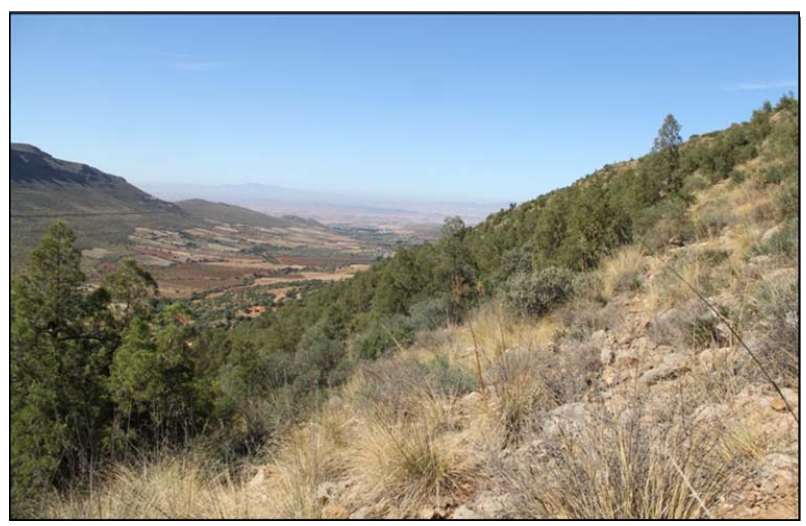

Figure 4. Degraded forest Tetraclinis articulata based atTameksalet-Moutas (south side). Photo. Babali B., October 2012. 
da Willd., Populus alba L., Salix pedicellata Desf., Juncus maritimus Lam., Hypericum perforatum L., Mentha rotundifolia L., Ficus carica L., Calamintha nepeta (L.) Savi, Potentilla reptans L., Zannichellia peltata Bertol., Groenlandia densa (L.) Fourr., Apium nodiflorum (L.) Lag, Arundo donax L., Dactylorhiza durandii (Boiss. \& Reuter) M. Lainz, Ranunculus ficaria L., Ranunculus aquatilis L., Ranunculus spicatus Desf. Sonchus maritimus L., Nerium oleonder L. Trachelium caeruleum L., Jasminum fruticans L., Vitis vinifera subsp. sylvestris (D.C. Gmelin) Hegi. Rorippa nasturtium-aquaticum (L.) Hayek (= Nasturtium officinale $\mathrm{R}$. Br.).

Riparian forests are dominant in this area; unfortunately the foresters do not take this fact into account in their statistics.

\subsection{The Scrub}

Over a large area of 680 ha, the scrubs are characterized and dominated by more xeric coppices depleted in forest and pre-forest species that occupy the land like thechamaephytes or nanaophanerophytes which prefer gradients and hot spots (southern slopes) belonging to Pistacio-Rhamnetalia Alaterni represented by Chamaerop shumilisvar. argentea Andrew Ampelodesmos mauritanicus (Poiret) Durand \& Schinz, Pistacia terebinthus L., Thymus munbyanus Boiss. \& Reuter, Fumana thymifolia (L.) Webb, Calicotome intermedia (Salzm.) C. Presl, Rhamnus lyciödes L.

Other taxa grow in an expansionary way after the fire e.g., Cistus ladanifer L., Cistus creticus L. and Cistus salvifolius L. and taxa characterized by their high regeneration such as thujaand evergreen oak that can participate in the formation of scrub landscapes [20].

\subsection{Lawns (Figure 5)}

"in the short-cycle crops adapted to use a fleeting resource, tolerance and/or need of light (light-demanding species) make them exclusive or preferential plants ofo-

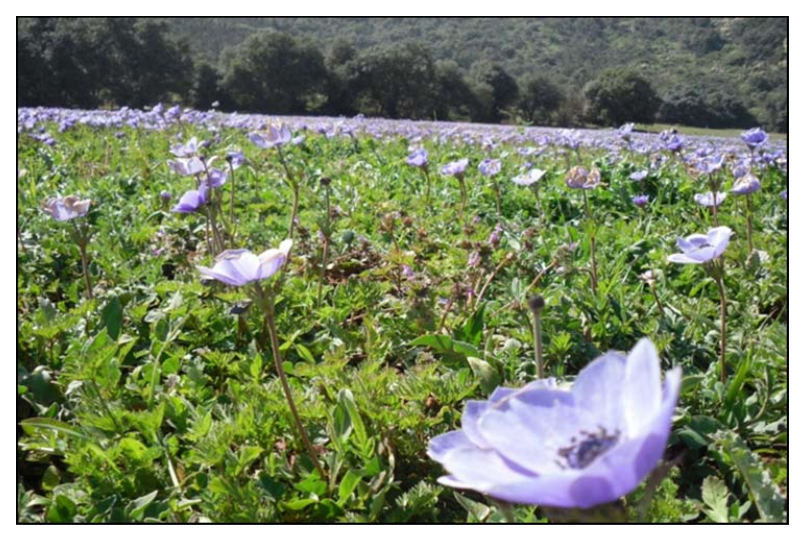

Figure 5. Annual plant lawn with Anemone coronaria before Moutas plain crops. Photo. Babali B., March 2011. ligotrophic dry grasslands or rocks exposed to wind and temperature extremes.” [32].

They are spread over about 106 hectares and dominated by annual species (Therophytes) caused by high anthropozoological action and further degradation (fire).

This group belongs to the Thero-Brachypodietea in general, is on calcareous substrata; characterized by Rhaponticum coniferum (L.) Greuter, Bombycilaena discolor (Pers.) Lainz, Reichardia tingitana (L.) Roth, Scorzonera laciniata L., Trifolium stellatum L., Ajuga iva var. pseudoiva subsp. Pseudoiva (DC.) Briq., Teucrium polium L., Ophrys lutea subsp. lutea (Cav.) Gouan, Carex halleriana Asso., Rumex bucephalophorus L., Briza maxima L. Vulpia ciliate Dumort...

List of vascular taxa listed by family in Tlemcenhunting reserve.

The list includes 322 species belonging to 72 families: Compositae (Asteraceae), Leguminosae (Fabaceae), Labiatae (Lamiaceae), Gramineae (Poaceae) and Orchidaceae (see palnches 1 - 2 in the annex).

$>$ ALLIACEAE

Allium Cupani Raf.

Allium roseum $\mathrm{L}$.

$>$ AMARYLLIDACEAE

Acis autumnalis (L.) Herb

=Leucojum autumnale L.

Narcissus cantabricus DC.

Narcissus serotinus $\mathbf{L}$.

Narcissus tazzeta $\mathbf{L}$.

Narcissus tazzeta subsp. pachybolbus (Dur.) Baker

Pancratium foetidum var. oranense Pomel

$>$ ANACARDIACEAE

Pistacia lentiscus $\mathbf{L}$.

Pistacia Terebinthus L.

$>$ APOCÉNACEAE

Nerium oleander $\mathrm{L}$.

$>$ ARACEAE

Arisarum simorrhinum Durieu

=Arisarum vulgare subsp simorrhinum (dur.) M.et W.

Biarum Bovei subsp. dispar (Schott.) Engler

$>$ ARALIACEAE

Hedera helix L.

$>$ ARECACEAE = PALMACEAE

Chamaerops humilis subsp. Argentea André.

$>$ ARISTOLOCHIACEAE

Aristolochia baetica L.

Aristolochia paucinervis Pomel

=Aristolochia longa subsp. paucinervis (Pomel) Batt.

$>$ ASPARAGACEAE

Anthericum liliago L. subsp. algeriense

Aphyllanthes monspeliensis L.

Asparagus acutifolius L.

Asparagus albus L.

ASPHODELACEAE 
Asphodelus ramosus L.

=Asphodelus microcarpus Salzm et Viv

$>$ ASPLENIACEAE

Ceterach officinarum Lamk.

=Asplenium ceterach $\mathrm{L}$.

$>$ BORAGINACEAE

Anchusa italica Retz.

=Anchusa azurea Mill.

Borago officinalis L.

Cerinthe gymnandra Gasparr.

=Cerinthe major subsp. gymnandra (Aspar.)M.

Cynoglossum cheirifolium L.

Echium vulgare L.

Neatostema apulum (L.) I.M. Johnston

=Lithospermum apulum (L.) Vahl.

$>$ CAMPANULACEAE

Campanula dichotoma $\mathrm{L}$.

Campanula rapunculus $\mathrm{L}$.

Trachelium caeruleum L.

$>$ CAPRIPHOLIACEAE

Lonicera implexa $\mathrm{L}$.

Viburnum tinus subsp. tinus $\mathrm{L}$.

$>$ CARYOPHYLACEAE

Dianthus cintranus Boiss. \& Reuter

=Dianthus gaditanus Boiss.

Dianthus serrulatus subsp. macranthus Maire

Paronychia argentea Lam.

Silene vulgaris (Moench) Garcke

=Silene inflata (Salisb.)Sm.

Silene latifolia subsp. Iatifolia Poiret

Silene ramosissima Desf.

Stellaria media (L.) Vill.

$>$ CISTACEAE

Cistus clusiiDunal.

Cistus creticus L.

=Cistus villosusL.

Cistus ladanifer subsp. mauritianus Pau \& Sennen

=Cistus ladaniferusL.

Cistus salvifolius L.

Fumana thymifolia (L.) Webb

Halimium umbellatum (L.) Spach

Helianthemum cinereum (Cav.) Pers.

Helianthemum helianthemoides (Desf.) Grosser

Helianthemum salicifolium (L.) Miller

$>$ COLCHICACEAE

Colchicum lusitanum Brot.

=Colchicum autumnale $\mathrm{L}$.

Merendera filifolia Camb.

$>$ COMPOSITAE = ASTERACEAE

Anacyclus pyrethrum (L.) Link

Atractylis cancellata L.

Bellis sylvestris Cirillo

Bombycilaena discolor (Pers.) Laínz

=Micropus bombycinus subsp discolor Lag.
Calendula arvensis $\mathbf{L}$.

Calendula suffruticosa Vahl

Carlina gummifera (L.) Less.

Catananche caerulea $\mathrm{L}$.

Centaurea pullata L.

Cichorium intybus $\mathbf{L}$.

Cirsium echinatum (Desf.) DC.

Dittrichia viscose (L.) Greuter

=Inula viscose (L.) Ait.

Echinops strigosus L.

Filago fuscescens Pomel

Glebionis segetum (L.) Fourr.

=Chrysanthemum segetum $\mathrm{L}$.

Helichrysum stoechas (L.) Moench

=EIichrysum stoechas (L.) DC.

Inula montana $\mathbf{L}$.

Mauranthemum paludosum (Poiret) Vogt \& Oberprieler

=Leucanthemum paludosum (Poiret) non Bar.

Pallenis maritima (L.) Greuter

=Asteriscus maritimus (L.) Less.

Pallenis spinosa (L.) Cass.

Phagnalon saxatile (L.) Cass.

Phagnalon sordidum (L.) Reichenb.

Reichardia tingitana (L.) Roth

Rhaponticum acaule (L.) DC.

Rhaponticum coniferum (L.) Greuter

=Leuzea conifera (L.) DC.

Scolymus grandiflorus Desf.

Scolymus hispanicus L.

Scorzonera laciniata $\mathrm{L}$.

Scorzonera undulata Vahl.

Senecio vulgaris $\mathbf{L}$.

Sonchus asper (L.) Hill

Sonchus maritimus L.

Staehelina dubia L.

Taraxacum obovatum (Willd.) DC.

$>$ CONVULVULACEAE

Convolvulus althaeoides $\mathrm{L}$.

Convolvulus arvensis $\mathbf{L}$.

Convolvulus cantabrica $\mathrm{L}$.

Convolvulus humilis Jacq.

Convolvulus tricolor $\mathrm{L}$.

$>$ CRASSULACEAE

Pistorinia breviflora subsp. intermedia (Boiss. \&

Reuter) Greuter \& Burdet

=Cotyledon breviflora (Boiss.) M.

Sedum sediforme (Jacq.) Pau

Sedum album L.

Umbilicus rupestris (Salisb.) Dandy

=Cotyledon umbilicus-veneris subsp. Pendulina (DC.) Batt.

$>$ CRUCIFERAE $=$ BRASSICACEAE

Alyssum simplex Rudolphi 
Biscutella didyma $\mathbf{L}$.

Erysimum grandiflorum Desf.

=Erysimum bocconei (all.) Pers.

Lepidium hirtum (L.) Sm.

Lobularia maritima (L.) Desv.

Nasturtium officinale $\mathrm{R}$. Br.

= Rorippa nasturtium-aquaticum (L.) Hayek

Raphanus raphanistrum $\mathrm{L}$.

Sinapis alba L.

Sinapis arvensis $\mathrm{L}$.

$>$ CUPRISSACEAE

Juniperus oxycedrus L. subsp. oxycedrus

Tetraclinis articulata (Vahl) Masters

Cupressus sempervirens $\mathrm{L}$.

CYPERACEAE

Carex halleriana Asso

Carex hispida Willd.

$>$ DIOSCOREACEAE

Diocorea communis (L.) Caddick \& Wilkin

=Tamus communis $\mathrm{L}$.

$>$ DIPSACACEAE

Cephalaria leucantha (L.) Roemer \& Schultes

Lomelosia stellata (L.) Raf.

=Scabiosa stellata L.

Sixalix atropurpurea (L.) Greuter \& Burdet

=Scabiosa atropurpurea $\mathrm{L}$.

$>$ ERICACEAE

Arbutus unedo L.

Erica arborea $\mathrm{L}$.

Erica multiflora L.

$>$ EUPHORBIACEAE

Euphorbia helioscopia L.

Euphorbia nicrceensis AlI.

Euphorbia squamigera Lois.

$>$ FAGACEAE

Quercus coccifera L. subsp. coccifera

Quercus faginea subsp. tlemcenensis (DC.) Maire et Weiller

=Quercus faginea subsp. broteroi (Coutinho) A. Camus

Quercus Ilex subsp. Ballota (Desf.) A. DC.

Quercus suber L.

$>$ GENTIANACEAE

Centaurium erythraea Raf.

=Centaurium umbellatum (Gibb.) Beck.

$>$ GÉRANIACEAE

Erodium moschatum (L.) L'Hér.

Geranium malviflorum Boiss. \& Reute

$>$ GRAMINEAE $=$ GRAMINACEAE $=$ POACEES

Aegilops geniculata Roth

Aegilops triuncialis L.

Ampelodesmos mauritanicus (Poiret) Durand \&

Schinz

$=$ Ampelodesma mauritanica (Poiret) Dur.et Sch.
Anisantha madritensis (L.) Nevski

$=$ Bromus matritensis $\mathrm{L}$.

Anisantha rubens (L.) Nevski

=Bromus rubens L.

Arundo donax L.

Avena sativa $\mathrm{L}$.

Avena sterilis $\mathbf{L}$.

Brachypodium sylvaticum (Huds.) P. B.

Briza maxima L.

Bromus hordeaceus L.

Cynosurus echinatus L.

Festuca coerulescens Desf.

Hordeum murinum L.

Lagurus ovatus $\mathbf{L}$.

Macrochloa tenacissima (L.) Kunth

=Stipa tenacissima $\mathrm{L}$.

Vulpia ciliata Dumort.

HYACINTHACEAE

Drimia maritima (L.) Speta

=Urginea maritima var. pancration (Stein.) Baker.

Drimia undulata Jacq.

=Urginea undulata (Desf.) Steinh. subsp typica $M$.

Leopoldia comosa (L.) Parl.

=Muscari comosum (L.) Mill.

Muscari neglectum Guss.

Oncostema peruviana (L.) Speta

=Scilla peruviana $\mathrm{L}$.

Ornithogalum algeriense Jord. \& Fourr

$=$ Ornithogalum umbellatum $\mathrm{L}$.

Ornithogalum narbonense $\mathrm{L}$.

=Ornithogalum pyramidalisauct. non L.

Prospero autumnalis (L.) Speta

=Scilla autumnalis $\mathrm{L}$.

Prospero obtusifolium (Poiret) Speta

=Scilla obtusifolia Poiret

Uropetalum serotinum (L.) Ker Gawl.

=Dipcadi serotinum (L.) Medik.

$>$ HYPERICACEAE

Hypericum perforatum $\mathrm{L}$.

Hypericum tomentosum subsp tomentosum $\mathrm{L}$.

> IRIDACEAE

Gladiolus italicus Mill

=Gladiolus segetum Ker.-Gawl.

Iris planifolia (Mill.) Dur. et Sch.

Iris xiphium L.

Moraea sisyrinchium (L.) Ker Gawl.

= Gynandriris sisyrinchium (L.) Parl.

Romulea bulbocodium (L.) Seb.et Maur.

$>$ JUNCACEAE

Juncus maritimus Lamk.

LABIATAE = LAMIACEAE

Ajuga chamaepitys (L.) Schreber

=Ajuga chamaephitis Schreb.

Ajuga iva subsp. iva (L.) Schreber 
Ajuga iva subsp. pseudoiva (DC.) Briq. var. pseudoIva

Ballota hirsutaBentham

Calamintha nepeta (L.) Savi

=Satureja calamintha subsp. Nepeta correct

Lamium amplexicaule $\mathrm{L}$.

Lavandula stoechas L. subsp. stoechas

Marrubium vulgare $\mathrm{L}$.

Mentha rotundifolia $\mathrm{L}$.

Nepeta multibracteata Desf.

Nepeta tuberosa subsp. reticulata (Desf.) Maire

Origanum vulgare subsp. Glandulosum (Desf.) Iestwaart

$=$ Origanum glandulosum Desf.

Phlomis crinita subsp. Mauritanica (Munby) Murb.

$=$ Phlomis crinita cav.

Phlomis herba-venti L.

Rosmarinus eriocalyx Jord. \& Fourr.

$=$ Rosmarinus tournefortiide Noé

Salvia verbenaca $L$.

Stachys ocymastrum (L.) Briq.

Teucrium fruticans $\mathrm{L}$.

Teucrium polium $\mathrm{L}$.

Teucrium pseudochamaepitys L.

Thymus munbyanus subsp. coloratus (Boiss. \&

Reuter) Greuter \& Burdet

$=$ Thymus ciliatus subsp. coloratus (B. \& R.) Batt.

$>$ LEGUMINOSAE $=$ FABACEAE

Anthyllis polycephala Desf.

Anthyllis vulneraria L.

Argyrolobium zanonii (L.) Link

$=$ Lotophyllus argenteus $\mathrm{L}$.

Astragalus caprinus subsp. caprinus.

=Astragalus caprinus subsp. Lanigerus (Desf.) $M$

Astragalus epiglottis $\mathbf{L}$.

Bituminaria bituminosa (L.) Stirton

$=$ Psoralea bituminosa $\mathrm{L}$.

Calicotome intermedia (Salzm.) C. Presl

=Calycotome villosa subsp. Intermedia (Salzm.) M.

Ceratonia siliqua L. (Césalpiniacées)

Coronilla scorpioides (L.) W.D.J. Koch Cytisus

arboreus subsp. baeticus (Webb) Maire

Cytisus villosus Pourret

=Cytisus triflorus L'Herit

Erophaca baetica (L.) Boiss.

$=$ Astragalus lusitanicus Lamk.

Genista ramosissima (Desf.) Poiret

= Genista cinerea subsp. ramosissima (Desf.) Maire

Genista tricuspidata subsp. Duriaei (Spach.) Batt.

Lathyrus latifolius L.

Lotus hispidus DC.

Medicago italica subsp. Tornata (L.) Emb. et Maire

Medicago polymorpha $\mathrm{L}$.

= Medicago hispida Gaertn.
Ononis biflora Desf.

Ononis pubescens $\mathrm{L}$.

Ononis spinosa $\mathrm{L}$.

Scorpiurus muricatus L.

Stauracanthus boivinii (Webb) Samp

=Ulex Boivinii Webbvar. webbianus (Cosson) Maire

Trifolium angustifolium $\mathrm{L}$.

Trifolium stellatum L.

Trifolium tomentosum L.

Vicia onobrychioides $\mathrm{L}$.

Vicia sativa L.

> LILIACEAE

Fritillaria lusitanica subsp. Oranensis (Pomel)

Valdés

=Fritillaria messanensis Raf. var. atlantica $\mathrm{M}$.

Gagea Durieui Pari.

Gagea granatelli subsp. chaberti Terracc.

Tulipa sylvestris subsp. australis (Link.) Pamp.

$>$ LINACEAE

Linum suffruticosum $\mathrm{L}$.

Linum tenue Desf.

Linum usitatissimum $\mathrm{L}$.

$>$ MALVACEAE

Lavatera trimestris $\mathrm{L}$.

Malope malachoides $\mathrm{L}$.

Malva sylvestris $\mathbf{L}$.

$>$ MORACEAE

Fucus carica L.

$>$ MYRSINACEAE $=$ PRIMULACEES

Anagallis arvensis $\mathbf{L}$.

Anagallis Anagallis monelli $\mathrm{L}$.

$>$ OLEACEAE

Jasminum fruticans $\mathbf{L}$.

Olea europea L. subsp. europaea

=Olea europea var. oleaster

Phillyrea angustifolia subsp. angustifolia $\mathbf{M}$.

Phillyrea latifolia L.

=Phillyrea angustifolia subsp. latifolia (L.)M.

$>$ ORCHIDACEAE

Aceras pyramidalis (L.) Reichenb

=Anacamptis pyramidalis (L.) L.C. Rich.

Anacamptis coriophora subsp. fragrans (Poll.) Bateman, Pridgeon \& Chase

= Orchis coriophora subsp. Fragrans (Poll.) G. Camus

Anacamptis morio subsp. tlemcenensis (Batt.) E.G.

Camus

Anacamptis papilionacea (L.) Bateman, Pridgeon \&

Chase

$=$ Orchis papilionacea $\mathrm{L}$.

Dactylorhiza durandii (Boiss.\& Reuter) M.Lainz

=Orchis elata subsp. Durandoi (B.et R.)

Himantoglossum hircinum (L.) Sprengel

=Himanthoglossum hircinum (L.) Spreng.

Himantoglossum robertianum (Loisel.) Delforge 
= Himanthoglossum longibracteatum (Biv.) Sch.

Ophrys atlantica Munby

Ophrys lutea subsp. Lutea (Cav.) Gouan

Ophrys speculum L.

Ophrys sphegifera Willd.

= Ophrys scolopax Cav. subsp. Apiformis

Ophrys subfusca (Reichenb. fil.) Haussknecht

=Ophrys lutea subsp. Subfusca (Rchb.) Batt.

Ophrys thenthredimifera Willd. subsp. Ficalhoana

Ophrys thenthredimifera Willd. subsp. Thenthredi-

mifera

Orchis anthropophora (L.) All.

= Aceras anthropophorum (L.) Ait.

Orchis italica Poiret

Orchis olbiensis Reuter.

= Orchis maculata subsp. Obliensis (Reut.) Asch. et Gr.

\section{$>$ OROBANCHACEAE}

Bartsia trixago L.

= Bellardia trixago (L.) All. (Scrophiliacées)

Odontites purpureus subsp. purpureus (Desf.) G. Don fil.

$=$ Odontites bolligeri E.Rico, L. Delgado \& Herrero in Rico et al. [33]

= Odontites purpureasubsp purpurea (Scrophiliacées)

Orobanche ramosa $\mathrm{L}$.

Orobanche variegata Wallr

Parentucellia latifolia (L.) Caruel (Scrophiliacées)

$>$ PAPAVERACEAE

Fumaria capreolata L. (Fumariacées)

Fumaria officinalis L. (Fumariacées)

Papaver hybridum L.

Papaver rhoeas $\mathbf{L}$.

Roemeria hybrida (L.) DC.

$>$ PINACEAE

Cedrus atlantica (Endl.) Carrière

$=$ Cedrus libanotica Link

Pinus halepensis Mill.

Pinus pinea $\mathrm{L}$.

$>$ PLANTAGINACEAE

Anarrhinum fruticosum subsp. fruticosum Maire (Scrophiliacées)

Antirrhinum majus L. (Scrophiliacées)

Globularia Alypum subsp. alypum L. (Globulariacées)

Linaria arvensis L. Desf. (Scrophiliacées)

Linaria triphylla (L.) Miller (Scrophiliacées)

Linaria tristis (L.) Miller (Scrophiliacées)

Plantago mauritanica Boiss. et Reut.

Plantago lagopus $\mathrm{L}$.

Plantago serraria $\mathrm{L}$.

$>$ POLYGALACEAE

Polygala monspeliaca L.

$>$ POLYGONACEAE
Rumex bucephalophorus $\mathrm{L}$.

$>$ POTAMOGETONACEAE

Groenlandia densa (L.) Fourr.

$=$ Potamogeton densusL.

$>$ RAFFLESIACEAE

Cytinus hypocistis subsp. clusiiNyman

$=$ Cytinus hypocistis subsp. kermesianus (Guss.)

Wettst.

Cytinus hypocistis subsp. hypocistis $\mathrm{L}$.

= Cytinus hypocistis subsp. ochraceus (Guss.) Wettst.

$>$ RENONCULACEAE

Adonis aestivalisL.

Anemone coronaria L.var. cyanea (Risso) Ardoino

Anemone palmata L.

Clematis cirrhosa $\mathrm{L}$.

Clematis flammula L. var. parviflora Pomel

Delphinium balansae Boiss. et Reut.

Ranunculus arvensis $\mathbf{L}$.

Ranunculus ficaria subsp. ficariiformis Rouy \&

Fouc.

Ranunculus gramineus $\mathbf{L}$.

Ranunculus macrophyllus Desf.

Ranunculus millefoliatus Vahl

Ranunculus paludosus Poiret

Ranunculus spicatus Desf.

Ranunculus aquatilis $\mathbf{L}$.

$>$ RESEDACEAE

Reseda alba subsp alba $\mathrm{L}$.

= Reseda alba subsp eu-alba L.

Reseda luteola $\mathrm{L}$.

Reseda Phyteuma subsp. collina (Gay) Batt.

$>$ RHAMNACEAE

Rhamnus alaternus L. subsp. alaternus

Rhamnus lycioides subsp. oleoides (L.) Jahand. \&

Maire

$>$ ROSACEAE

Crataegus monogyna Jacq.

= Crataegus Oxyacantha subsp. monogyna (Jacq.)

Rouy et Camus

Potentilla reptans $\mathrm{L}$.

Rosa canina $\mathrm{L}$.

Rubus ulmifolius Schott

Sanguisorba minor Scop.

$>$ RUBIACEAE

Rubia peregrina subsp. Peregrina $\mathrm{L}$.

$>$ RUSCACEAE

Ruscus aculeatus L.

$>$ RUTACEAE

Ruta angustifolia Pers.

$=$ Ruta chalepensis subsp. angustifolia (Pers.) P. Cout

SALICACEAE

Populus alba L.

Salix pedicellata Desf.

SANTALACEAE 


\section{Osyris quadripartita Decne \\ SAXIFRAGACEAE \\ Saxifraga globulifera Desf. \\ = Saxifraga globulüera Desf. \\ $>$ SCROPHILIACEAE \\ Scrofularia laevigataVahl \\ Scrophularia canina L. \\ Verbascum blattaria L. \\ SINOPTERIDACEAE \\ Cheilanthes acrostica (Balb.) Tod. \\ SMILACACEAE}

Smilax aspera L. var. Altissima Moris \& De Not.

Smilax aspera L. var. genuina $\mathrm{L}$.

$>$ THYMELAEACEAE

Daphne gnidium L.

$>$ THYPHACEAE

Typha angustifolia $L$.

$>$ UMBELLIFERAE = APIACEAE

Ammoides pusilla (Brot.) Breistr.

$=A$. verticillata (Desf.) Briq.

Apium nodiflorum (L.) Lag

= Helosciadium nodiflorumLag.

Bupleurum rigidum $\mathrm{L}$.

Daucus carota L.

Eryngium tricuspidatum L.

Eryngium triquetrum Vahl

Ferula communis L.

Thapsia garganica $\mathbf{L}$.

$>$ VALÉRIANACEAE

Fedia cornucopiae (L.) Gaertn

Valeriana tuberosa $\mathrm{L}$.

Valerianella discoidea (L.) Loisel.

= Valerianella coronata subsp. discoidea Lois.

$>$ VERBENACEAE

Verbena officinalis $L$.

VITACEAE = AMPELIDACEA

Vitis vinifera subsp. sylvestris (C.C. Gmelin) Hegi

ZANNICHELLIACEAE

Zannichellia peltata Bertol.

\section{CONCLUSION AND PERSPECTIVES}

One can not speak of plant diversity, of the Moutasreserve, without taking the relative change in the climate of the western part of Algeria into consideration. That's how the phylogenetic potential began a regressive evolution. This latter is accentuated by an increasingly strong anthropo zoological pressure. These ecosystems are fragile and complex and must be tackled in a comprehensive manner. There are many achievements in phytoecology and plant systematics, but little information/data is available regarding in particular the western part of Algeriaendemic species.

Faced with this alternative we insist on developing/expanding plants (aromatic/medicinal and others) sincethe hunting reserve has a wealth of landscapes and acts as a refuge for sensitive and/or endangered species as Origanum, Ammoides e.g.

Today, we are moving towards a bank of botanical data to monitor this floristic cover which is close to the ecological break (environmental stress).

For about 30 years we have gone through this area, the changes are significant and we see before our eyes that the regressive evolution has begun. This observation is linked to southern species which are moving to north of Tlemcen Mounts.

\section{REFERENCES}

[1] Blondel, J., (2002) Problématique de la forêt méditerranéenne. Hors Scin, 1.

[2] Gruber, M. and Sandoz, H. (1995) Inventaire floristique et phytoécologique du parc du Jarret-la Ravelle (Marseille, Bouches-du-Rhône, France). Bulletin de la Société Linnéenne de Provence, 46, 105-118.

[3] Véla, E., Hill B. and S. Della Casa (1999) Liste des plantes vasculaires du département des Bouches-duRhône (France). Bulletin de la Société Linnéenne de Provence, 50, 115-201.

[4] Hill, B. and véla, E. (2000) Mise à jour de la liste des plantes vasculaires du département des Bouches-duRhône. Bull. Bulletin de la Société Linnéenne de Provence, 51, 71-94.

[5] Ibn Tattou, M. and Fennane, M. (1989) Aperçu historique et état actuel des connaissances sur la flore vasculaire du Maroc. Bulletin Institut Scientifique de Rabat, 13, 85-94.

[6] Bouazza, M., Mahboubi, A., Loisel, R. and Benabadji, N. (2001) Bilan de la flore de la region de Tlemcen (OranieAlgérie), forêt méditerranéen, 2, 130-136.

[7] Benabadji, N., Benmansour, D. and Bouazza, M. (2007) La flore des monts d'Ain Fezza dans l'Ouest algérien, biodiversité et dynamique. Sciences \& Technologie, 26, 47-59.

[8] Mesli, K., Bouazza, M. and Godron, M. (2008) Ecological characterization of the vegetable groupings of the mounts of Tlemcen and their facies of degradation (west-Algeria). Environmental Research, 2, 271-277.

[9] Letreuch-Belarouci, A., Medjahdi, B., Letreuch-Belarouci, N. and Benabdeli, Kh. (2009) Diversité floristique des subéraies du parc national de Tlemcen. Acta Botanica Malacitana, 34, 77-89.

[10] Medjahdi, B., Ibn Tattou, M., Barkat, D. and Benabedli, Kh. (2009) La flore vasculaire des monts des Traras. Acta Botanica Malacitana, 34, 57-75.

[11] Anonymous-Bulgarie (1988) Projet d'aménagement cynégétique de la réserve de chasse Moutas-wilaya de Tlemcen. Lescomplekt-engineering, 4, 99.

[12] Braun-Blanquet, J. (1951) Les groupements végétaux de la France méditerranéenne., CRNS, Paris, 297.

[13] Quézel P. and Santa S. (1962-1963) Nouvelle flore d’Algérie et des régions désertiques méridionales. CRNS, 
Paris (FR), Tome I: 1-565, Tome II: 566-1170.

[14] Battandier, A. and Trabut, L. (1888-1890) Flore d'Algérie (Dicotylédones). 860.

[15] Valdés, B., Rejdali, M., Kadmiri, A.A.E., Jury, S.L. and Montserrat, J.M. (2002) Catalogue des plantes vasculaires du Nord du Maroc incluant des clés d'ídentification. Consejo Superior d'Investigaciones Científicas, Biblioteca de ciencias, Madrid, Vol. I and II, 1007.

[16] Blanca, G., Cabezudo, B., Cueto, M., Fernández López, C. and Morales Torres, C. (2009) Flora Vascular de Andalucía Oriental, Consejería de Medio Ambiente, Junta de Andalucía, Sevilla.

[17] Maire, R. (1952-1987) Flore de l'Afrique du Nord (Maroc, Algérie, Tunisie, Tripolitaine, Cyrénaïque et Sahara). Le Chevalier, Paris. Vol. 1-16, 5559.

[18] Dobignard, A. (2008) Index, and synonymous bibliographic flora of North Africa, in Press.

[19] Dahmani-Megrerouche, M. (1996) Diversité biologique et phytogéographique des chênaies vertes d'Algérie, Ecologia Mediterranea, 22, 19-38.

[20] Quezel, P. and Medail, F. (2003) Ecologie et biogéographie des forêts du bassin méditerranéen. Elsevier, Paris, 592.

[21] Laribi, M., Derridj, A. and Acherar, M. (2008) Phytosociologie de la forêt caducifoliée à chêne zéen (Quercus canariensis willd.) Dans le massif d'Ath Ghobri-Akfadou (grande Kabylie, Algérie). Fitosociologia, 45, 1-15.

[22] Messaoudène, M., Tafer, M., Loukkas, A. and Marchal, R. (2008) Propriétés physiques du bois de chêne zéen de la forêt des Aït Ghobri (Algérie). Bois et Forêts des Tropiques, 298, 37-48.

[23] Zine El Abidine, A. (1988) Analyse de la diversité phytoécologique des forêts du chêne zeen (Quercus faginea Lamk.) Au Maroc. Bulletin Institut Scientifique de Rabat, 12, 69-77.

[24] Alcaraz, C. (1989) Contribution à l'étude des groupements à Quercus ilex et Quercus faginea subsp. Tlemcenensis des monts de Tlemcen (Algérie). Ecologia Medi- terranea, 15, 15-32.

[25] Prodon, R., Fons, R. and Peter, A.M. (1984) L'impact du feu sur la végétation, les oiseaux et les micromammifères dans diverses formations méditerranéennes des Pyrénées Orientales: Premiers résultats. Revue d'écologie, Terre et Vie, 39, 128-158.

[26] Amandier, L. (2004) Le comportement du Chêne-liège après l'incendie conséquences sur la régénération naturelle des subéraies. Actes du coloque-VIVexpo 2004: Le chêne-liège face au feu, 1-18.

[27] Schaffhauser, A., Curt, T., Véla, E. and Tatoni, T. (2012) Feux récurrents et facteurs environnementaux façonnent la végétation dans les boisements à Quercus suber L. Et les maquis, C.R. Biologies, 335, 424-434. doi:10.1016/j.crvi.2012.04.006

[28] Hadjadj-Aoul, S. (1995) Les peuplements du Thuya de Berbérie (Tetraclinis articulata (Vahl) Master) en Algérie: phytoécologie, syntaxonomie et potentialités sylvicoles. Thèse d'Etat, Université Aix-Marseille III, Aix-en-Provence and Marseille, 250.

[29] Hadjadj-Aoul, S., Chouieb, M. and Loisel, R. (2009) Effet des facteurs environnementaux sur les premiers stades de la régénération naturelle de Tetraclinis articulata en Oranie, Ecologia Mediterranea, 35, 19-30.

[30] Quézel, P. (2000) Réflexions sur l'évolution de la flore et de la végétation au Maghreb méditerranéen. Ibis Press, Paris, 117.

[31] Quezel, P. and Medail, F. (2003) Valeur phytoécologique et biologique des ripisylves méditerranéennes. Forêt Méditerranéenne, 3, 231-248.

[32] Véla, E. (2002) Biodiversité des milieux ouverts en région méditerranéenne. Le cas des pelouses sèches du Luberon (Provence calcaire). Phd thesis. University Aixmarseille III, Aix-en-Provence and Marseille, 383.

[33] Rico, E., Delgado, l. and Herrero, A. (2008) Reassessing the Odontites purpureus group (Orobanchaceae) from South-East Spain and North-West Africa. Botanical Journal of the Linnean Society, 158, 701-708. 


\section{Appendix}

Planche 1. Board color (phot. B. Babali).

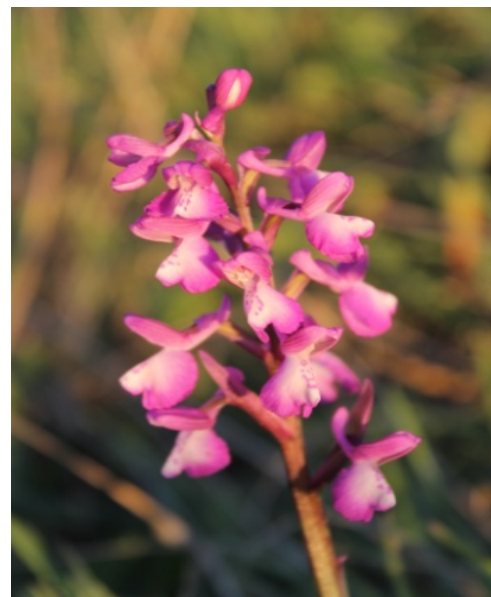

Anacamptis morio subsp. tlemcenensis (Batt.) E.G. Camus

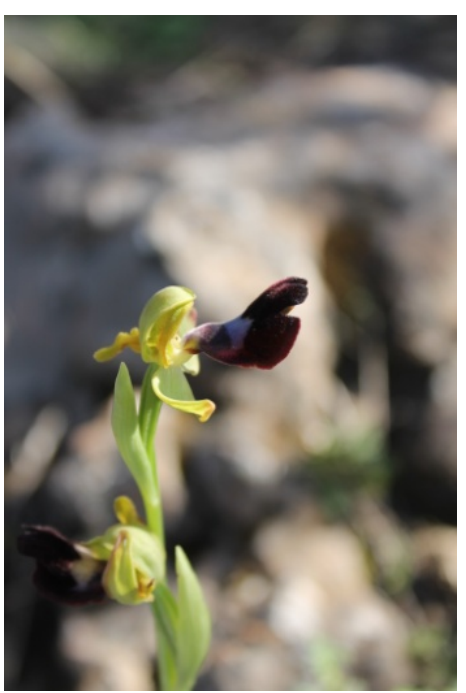

Ophrys atlantica Munby

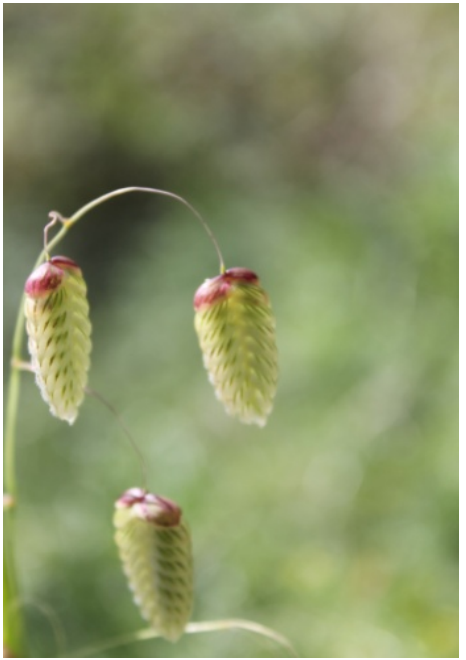

Briza maxima L.

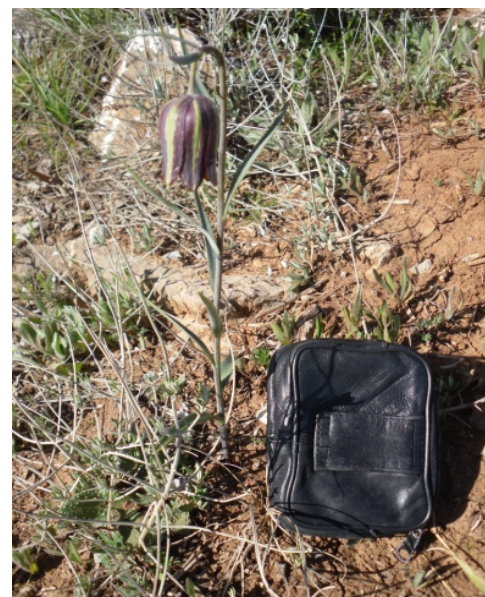

Fritillaria lusitanica subsp. Oranensis (Pomel) Valdés

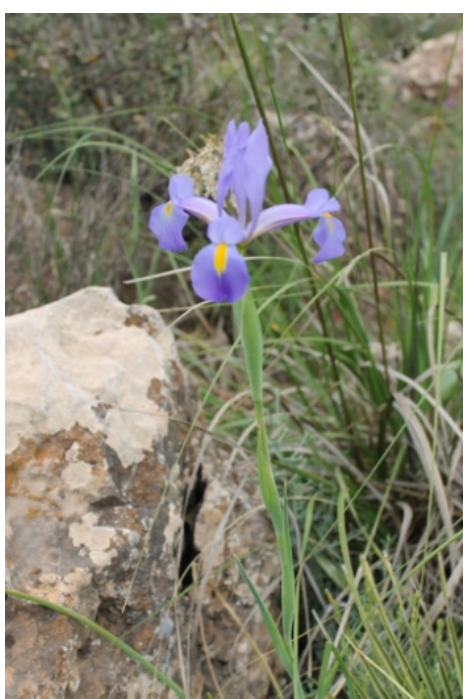

Iris xiphium $L$.

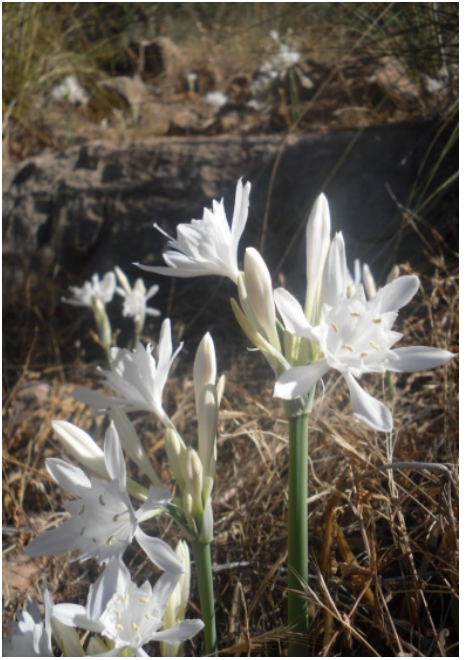

Pancratium foetidum var. oranense Pomel

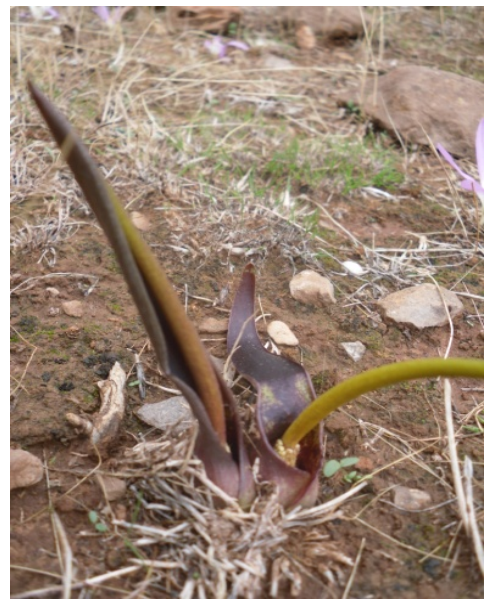

Biarum Bovei subsp. dispar (Schott.) Engler

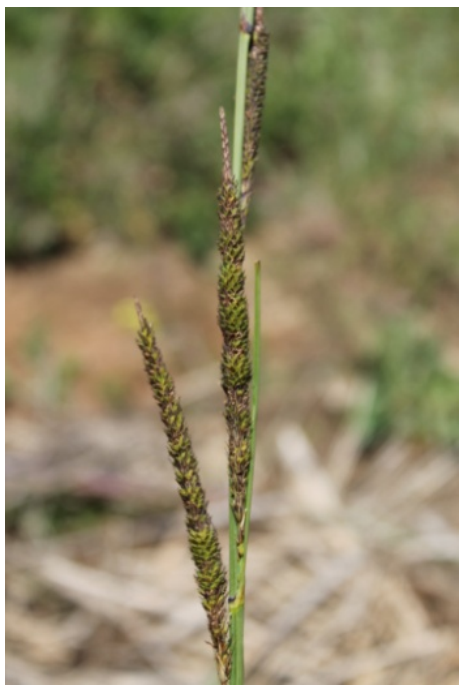

Carex hispida Willd.

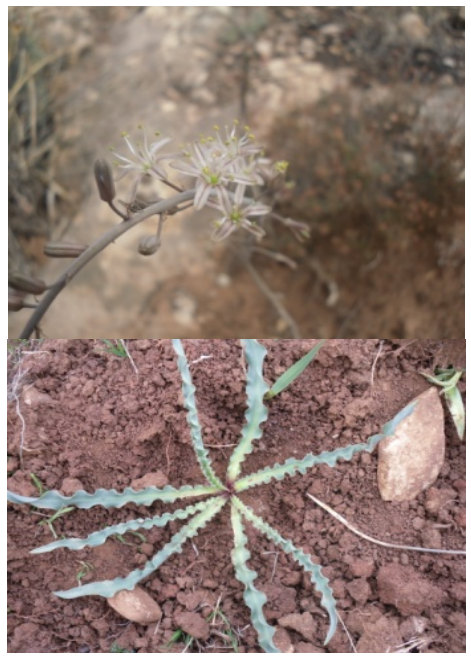

Drimia undulata Jacq. 
Planche 2. Board color (phot. B. Babali).

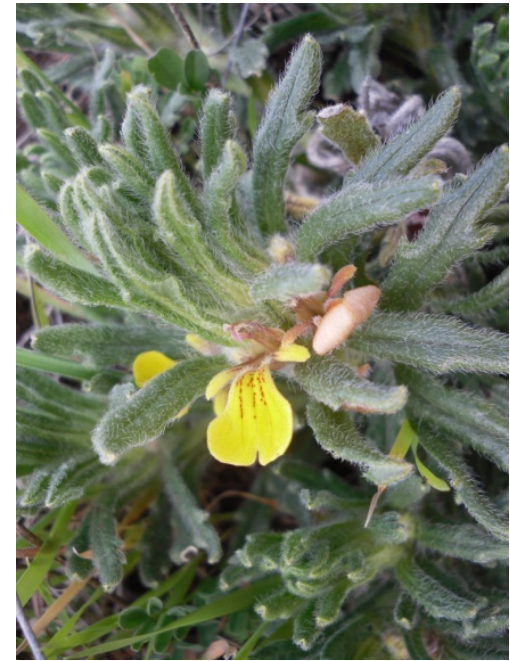

Ajuga iva subsp. pseudoiva (DC.) Briq.

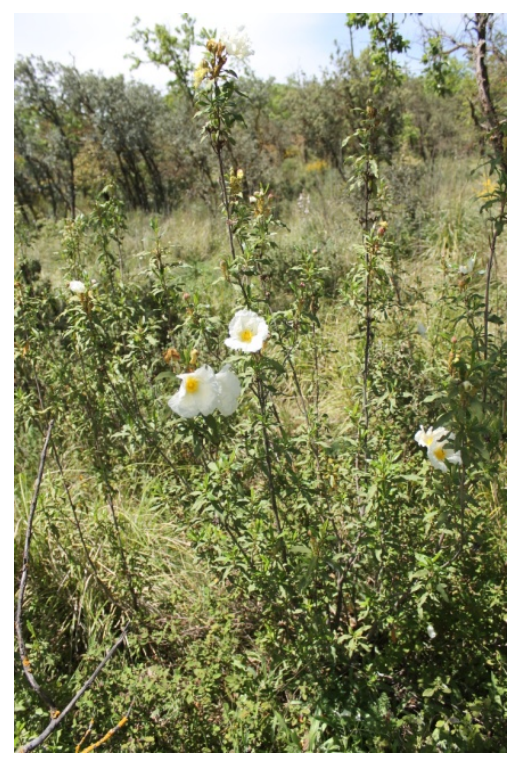

Cistus ladanifer subsp. Mauritianus Pau \&Sennen

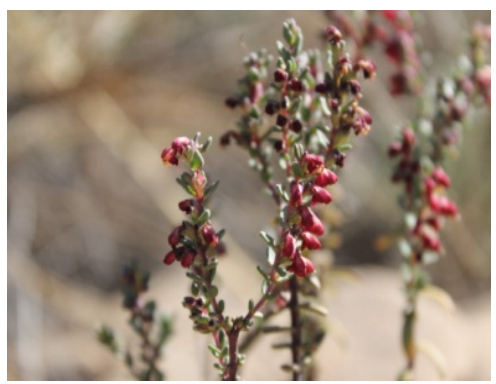

Odontites purpureus subsp. purpureus (Desf.) G. Don fil.

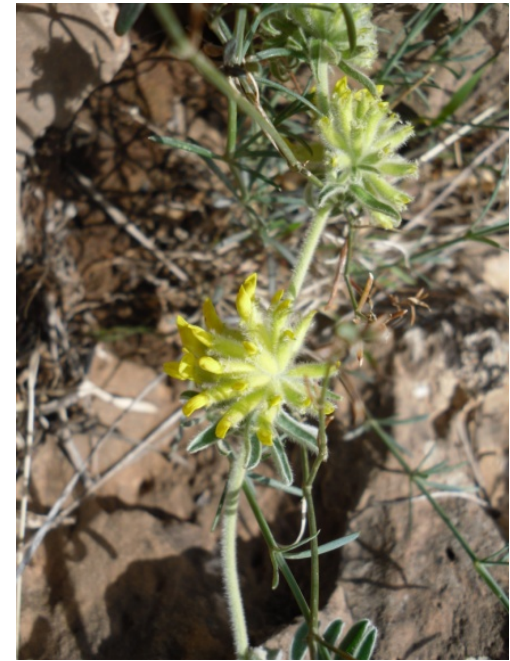

Anthyllis polycephala Desf.

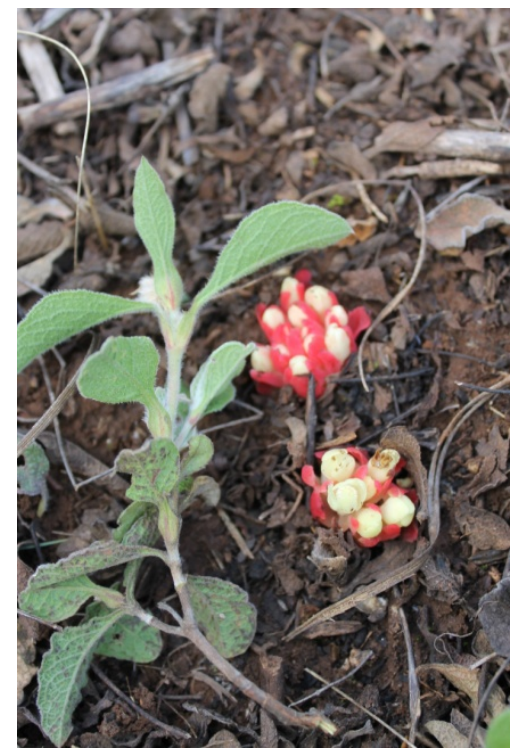

Cytinus hypocistis subsp. clusii Nyman + Cistus villosus Pourret

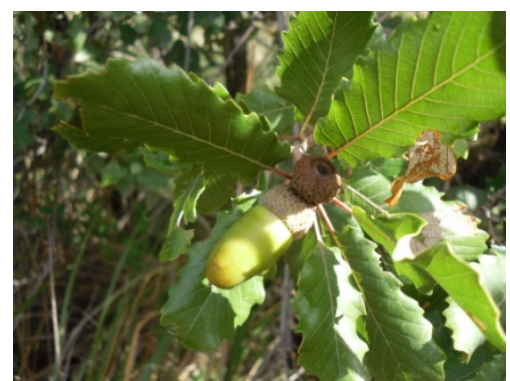

Quercus faginea subsp. tlemcenensis (OC.) Maire et Weiller

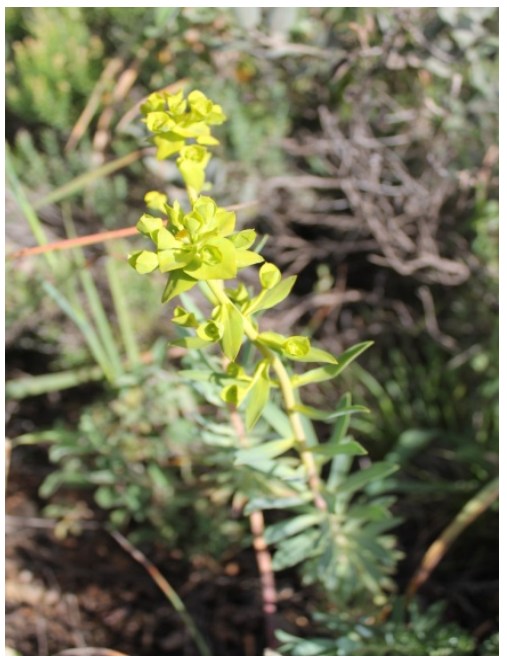

Euphorbia nicræensis AlI.

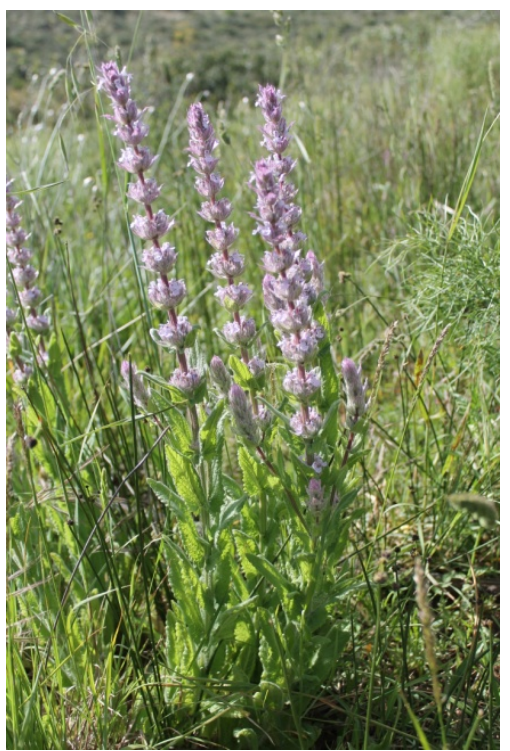

Nepeta tuberosa subsp. reticulata (Desf.) Maire

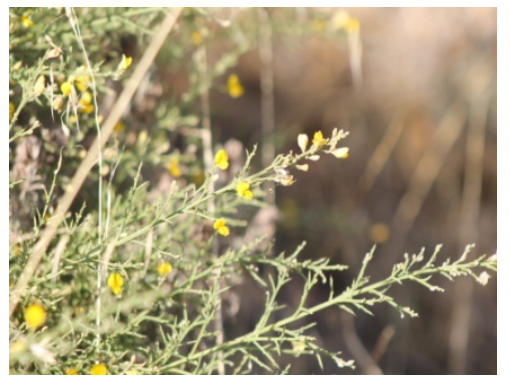

Stauracanthus boivinii

(Webb) Samp 